
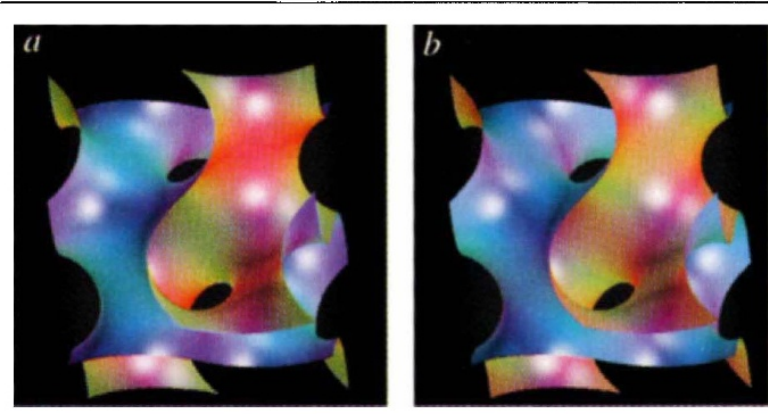

FIG. 3a, The gyroid, a triply periodic, space-dividing minimal surface that contains no lines and has no reflective symmetries. $b$, Almost identical, the solution of $\sin x \cos y+$ $\sin y \cos z+\sin z \cos x=0$ (Images courtesy of James T. Hoffman, MSRI.)

curvature surfaces are found as dividing surfaces in many materials ${ }^{8}$.

To simplify calculations, materials scientists and crystallographers have substituted for the gyroid - and for most other triply periodic minimal surfaces - the locus of solutions to a single trigonometric equation. For example, the solution to $\sin x \cos y+\sin y \cos z+\sin z \cos x=0$ is, visually, amazingly close to the gyroid (Fig. $3 b)$. Such 'zero-set surfaces' are useful in describing dividing surfaces where both materials have equal volume fraction; perturbations of them (with the zero replaced by a small constant) may be useful where the materials are unequal ${ }^{9}$.

These functions are not found by chance; they come from choosing an appropriate lowest-order term from the Fourier series of an electrostatic potential function coming from a charge distribution with the desired space-group symmetry. But there is as yet no explanation as to why this works, and in some cases it does not work very well. Moreover, these zero-set surfaces are not minimal surfaces, yet properties of minimal surfaces are claimed for them when convenient and ignored when they are troublesome.

Elser's surface ${ }^{1}$ is the union of three zero-set surfaces. It is not minimal and there may not be a minimal surface close to it, in the sense that the gyroid is close to the zero set of the equation above. Moreover, the conversion of a rotational motion to a translation, the Archimedean-screw property, is not a property of this surface at all, but a property of a family of zero-set surfaces, considered as deformations of the original one (two examples are shown in Fig. 1). None of them are minimal and no two of them are congruent. They only have a weak form of handedness when taken as a family.

For a mathematician, this is troublesome. Consider that the gyroid has only recently been shown by rigorous mathematical means to be a space-dividing surface $^{10}$. That is an important result, even though the evidence of its validity was already overwhelming from carefully generated models and computer images. For geometers, simulating a surface on a computer is a step along the way to understanding it mathematically, whereas a materials scientist has no use for an abstract surface without the ability to visualize it.
Archimedes had something relevant to say about this situation. Discussing the difference in mathematics between means of discovery and methods of proof, he wrote that "...certain things became clear to me by a mechanical method, although they had to be demonstrated by geometry afterwards because their investigation by the said method did not furnish an actual demonstration. But it is of course easier, when we have previously acquired, by the method, some knowledge of the questions, to supply the proof than it is to find it without any previous knowledge..."

In thought-experiments and in real ones, Archimedes applied mechanics the law of the lever in particular - to discover geometric relationships. He then tried to prove them by more formal means, and often he succeeded. What appears to be happening in materials science today can be viewed as an inversion of this process: new physical structures are being discovered by the sometimes very loose application of differential geometry. Their validation depends on whether these structures organize and predict observable phenomena, not on whether or not the theory was used correctly from a mathematical standpoint.

Materials science and mathematics may be immiscible, but with computer simulations and computer graphics as surfactant, they are interacting in unusual and productive ways.

David Hoffman is at the Mathematical Sciences Research Institute, Berkeley, California 94720, USA.

\footnotetext{
Elser, V. Phil. Trans. R. Soc. Lond. A 354, 2071-2075 (1996)

2. Pansu, B. \& Dubois-Violette, E. J. Phys. Colloq. 57, C7 281-297 (1990)

Thomas, E. L., Anderson, D. A., Henkee, C. S. \& Hoffman, D. Nature 334, 598-601 (1988).

4. Schwarz, H. A. Gesamm. Math. Abh. ISpringer, Berlin, 1890).

5. Hildebrandt, S. \& Tromba, A. The Parsimonious Universe 197-202 (Springer, New York. 1996).

6. Schoen, A. NASA Tech. Note TN D-5541 (1970) 7. Karcher, H. \& Poithier, K. Phil. Trans. R. Soc. Lond. A 354, 2077-2104 (1996).

8. Hajduk, D. A. et al. Macromolecules 27, 4063-4075 (1994).

. Lambert, C., Radzilowski, L. \& Thomas, E. Phil. Trans. $R$ Soc. Lond. A 354, 2009-2024 (1996)

10. Grosse-Braukmann. K. \& Wohlgemuth, M. preprint SFB 256 (Univ. Bonn, 1995).

11. Archimedes On the Method (transl. Heath, T. L.) p.2
} (Cambridge Univ. Press, 1912).

\section{Olbers' uproar}

OLBERS' astronomical brightness paradox argues that if the Universe is infinite and evenly populated with stars, the night sky should be infinitely bright. Daedalus is applying an acoustic form of the argument to parties and social gatherings. Divide the area round any listener into annular shells. Shells of larger radius are more distant, but contain more people. Accordingly, with an acoustically reflective floor and ceiling, each shell will radiate to the central listener an added equal increment to the hubbub. An infinite party would therefore be totally deafening.

As with Olbers' paradox, there are several ways out. Sound has a finite speed, so sufficiently distant voices will not reach the listener until the party has been going on indefinitely long. Or the party-goers might form clusters which are themselves clustered, and so on, defeating the summation. The party might even be expanding.

Most parties, however, are even noisier than Olbers would predict. Each talker speaks ever louder to overcome the hubbub of the others, who react by speaking louder still. A further aggravating factor is background music. Initially it serves a useful purpose; it masks the occasional awkward silence when the few early arrivals all happen to fall silent together. Thereafter, it merely stokes the acoustic catastrophe.

Pop radio stations use a constant-level mixing system, which fades the music for the disc jockey's voice. In a restaurant or a party, such 'fill-in' music would mask the silences, yet fade towards zero intensity as the crowd noise rose. But to prevent acoustic catastrophe, further rises in noise level would require music of negative intensity.

So Daedalus is inventing it. A set of uncorrelated conversations contains all frequencies. Subtract the frequencies you don't want, and you could get any desired tone or musical chord. Daedalus's 'Negative Muzak' is created by a set of fast-acting, damped acoustic cavities around the room, which absorb a chosen programme of frequencies. In a roomful of talkers, they will extract just that sequence of frequencies from the hubbub which leaves behind the programmed tune. With luck, the perfect subtraction of just one frequency from white noise will imply that frequency to the ear; but for the strongest effect, more complete subtractions may be needed. Negative Muzak will provide background musical entertainment while subtracting from the acoustic hubbub rather than adding to it. Frustrating Olbers, it will bring welcome civility to restaurants and parties everywhere.

David Jones 